\title{
The Expanded Programme on Immunisation in South Africa: A story yet to be told
}

\author{
N R Dlamini, ${ }^{1}$ BSc, MB ChB, MMed (Paed); P Maja, ${ }^{2}$ BA Hons \\ ${ }^{1}$ Child, Youth and School Health: National Department of Health, Pretoria, South Africa \\ ${ }^{2}$ Communication and Stakeholder Engagement: National Department of Health, Pretoria, South Africa
}

Corresponding author: P Maja (majap@health.gov.za)

During the past two decades, immunisation has saved millions of lives and prevented countless illnesses and disabilities in South Africa (SA). However, vaccine-preventable diseases are still a threat. A vaccine-preventable disease that might lead to a 1- or 2-week illness in an adult, could prove deadly for infants, children or elderly people. Vaccination protects oneself and one's family. For example, adults are the most common source of pertussis (whooping cough) infection in infants, which can be deadly for the latter. This article demonstrates the commitment of the SA government to immunisation, highlights key milestones of the Expanded Programme on Immunisation (EPI) and dispels the myth that the EPI in SA is in shambles.

S Afr Med J 2016;106(7):675-677. DOI:10.7196/SAMJ.2016.v106i7.10956

Recently, the South African Medical Journal published an article entitled 'Vaccines: SA's immunisation programme debunked. ${ }^{[1]}$ The article extensively quotes someone who has resigned from the Department, and to give credence to the avalanche of negativity about the country's Expanded Programme on Immunisation (EPI), the former employees of the Department are posted as the top National Department of Health (NDoH) EPI managers with substantial years of experience and, supposedly, expertise.

When a journalist publishes a story of the views of the former employees and does not seek the voice of those who are still serving, one will not be able to know whether what is reported is fact or sour grapes. Good journalism is balanced journalism - giving the protagonists, so to speak, equal opportunity to tell their versions of the story.

The resignation of the two employees did not create a crisis for the EPI for two reasons: (i) the implementation of EPI is done at provincial level, and the NDoH's responsibility is to develop policies and monitor their implementation; and (ii) the supervisors of these two former employees have years of experience, not only in immunisation but child health as a whole, which is more than just basic EPI. This is of paramount importance because integration is needed for efficiencies and cost-effectiveness in the delivery of health programmes. Their years of experience certainly contribute to institutional memory.

Staffing of the EPI unit at the national office has been prioritised. Posts were advertised following the resignation of the two employees, interviews have been conducted and the process of appointment is being finalised. Some of the incumbents have taken up the posts and others are still going through the process. All the posts will be filled, including that of the medical officer.

\section{Human resources for health}

With the Sustainable Development Goals (SDGs), the global community sees greater than ever consensus about the crucial role that the health workforce plays in realising goals to achieve universal health coverage. Qualified health workers, trained to work in effective teams within and across professional cadres to address the biomedical and social determinants of health, are critical to achieving health goals. While great progress has been made in maternal and child health and HIV/AIDS, continued shortages of adequately trained health workers raise the question of whether health professional education (HPE) systems are producing the health workforce needed to meet outstanding and emerging global health challenges.

There is recognition by the World Health Organization (WHO) and other health scientists across the world that the training of health professionals does not meet the health needs of the 21st century. ${ }^{[2,3]}$ Training of health professionals is based on outdated, fragmented, and content-oriented curricula that produce graduates with narrow contextual understanding and insufficient knowledge, skills, and competencies to understand social and other determinants of health and burden of disease. This outdated training has led to: poor teamwork and inadequate collaboration within and across health professional cadres; episodic encounters with patient illnesses rather than continuous and holistic healthcare; a predominant hospital orientation at the expense of primary care; an imbalance between health workforces and health needs; and weak leadership in improving health system performance. ${ }^{[4]}$

The achievement of the health-related objectives of the National Development Plan (NDP) and SDGs is contingent on a skilled, competent, multiprofessional and interprofessional health workforce working synergistically to address the quadruple burden of disease. ${ }^{[5]}$

The NDoH is working towards a health workforce that has the right skills, and works in the right place at the right time to provide the right services to the right people. ${ }^{[4]}$ Above all, the Department is encouraging integrated teams that are comprised of team players. Persons who elevate themselves beyond team work often find it difficult to fit into the new culture.

\section{The effectiveness of the EPI in South Afficica (SA)}

Scientific literature on the effectiveness of EPI in SA is prolific and includes reports of studies done by independent researchers. ${ }^{[6-8]}$

Madhi $^{[9]}$ has reported that in SA under-5 childhood deaths increased from 74753 in 2000, peaked at 89418 in 2005 and declined to 47409 in 2013. He attributes this to the introduction of the EPI. 
Immunisation averts some 2.5 million premature deaths a year according to estimates and protects millions of children from illness and disability. Parents and children attending a clinic in SA are among those today who will reap the benefits of routine immunisation from the first days of life. ${ }^{[9]}$

South Africa now has 11 antigens in the EPI schedule, including the most recently introduced human papillomavirus (HPV) vaccine for protection of young girls against cancer of the cervix that may affect them later in life. The immunisation programme has come a long way since it was launched in 1974, when there were only six vaccines in the schedule: diphtheria, pertussis and tetanus (DPT), measles, polio and Bacillus Calmette-Guérin (BCG) for protection against tuberculosis. ${ }^{[6]}$

The EPI in SA has made significant achievements in the last few decades, including:

- Polio has been eliminated in SA, with a last case found in 1989.

- Other conditions that are under control are diphtheria and pertussis, commonly known as whooping cough.

- SA has been in the forefront of introducing new vaccines. In 1995 the country introduced hepatitis B (hep B) vaccine. In 1999 Haemophilus influenzae type $\mathrm{b}$ (Hib) vaccine was introduced. 2009 saw the introduction of pneumococcal vaccine (PCV) and rotavirus vaccine (RV), and most recently in $2014 \mathrm{HPV}$ to prevent cervical cancer. On the continent, SA has been one of the first countries to introduce these vaccines - all fully funded by government. The programme continues to improve as evidenced by the fact that early in 2015 pentavalent vaccine was phased out and replaced by the more baby-friendly hexavalent so that the baby is only pricked once to receive six antigens.

When SA introduced the PCV and RV, the Department asked the National Institute for Communicable Diseases (NICD) to establish a surveillance system and to study the impact of these new vaccines on incidence of pneumonia and diarrhoea. The NICD reports that there has been a $>40 \%$ reduction in pneumococcal diseases across all ages. The greatest decline is among children $<2$ years of age, the age group that carries the highest levels of the disease. In addition the NICD found that there was a $34 \%$ decline in pneumococcal disease in people $\geq 45$ years old. Besides the impact of vaccine on pneumonia, the NICD reported that the vaccine has an effect on antimicrobial resistance - which is a global problem and if not urgently addressed can reduce the effectiveness of the antibiotics that are currently used. The pneumococcus vaccine contributes to a significant decline in penicillin, ceftriaxone (Rocephin) and multidrug-resistant strains of pneumococcus. ${ }^{[7,10]}$

Similar surveillance on the impact of the RV shows that the vaccine has resulted in a $40 \%$ reduction in the hospitalisation of children with diarrhoea (attributable to all causes) and 60\% reduction in hospitalisations from diarrhoea that is caused by rotavirus.

In 2014 the Department of Health introduced the HPV for all girls in grade 4 who are $\geq 9$ years old. The HPV vaccine protects from cancer of the cervix when they are older women. It is known that cancer of the cervix is the most common cancer among women in SA and predominantly affects black women, at an increasingly younger age. Cancer of the cervix has debilitating complications and often severely compromises the quality of life of those affected before they eventually succumb to it. It is estimated that 6000 women a year in SA contract cervical cancer and about half of them die from the disease.

SA was the first country on the African continent to introduce the two new vaccines. PCV has now been introduced in other countries in Africa such as Kenya and Rwanda that are supported by the Global Alliance for Vaccines and Immunisation (GAVI). SA remains the only country in the WHO Africa region that selffinances vaccines. ${ }^{[9]}$

The introduction of PCV and RV is a significant public health milestone for the country and the continent. Furthermore, it points to the leadership role that SA has taken on the continent with the introduction of new and underutilised vaccines, dating back to 1995 with the introduction of hep B and, in 1999, Hib vaccines.

\section{Sound decision-malking process}

Given the complexity of the introduction of new vaccines and that such decisions relate to an important public health programme, it is crucial that these decisions which establish immunisation policies are well informed, unbiased, evidence based and locally relevant. This has been the basis of the formation of national immunisation technical advisory groups (NITAGs) in many countries, particularly in developed countries where these groups are well established. SA has a functional NITAG, referred to as the National Advisory Group on Immunisation (NAGI). NAGI is effective as a source of information and an advisory body to the $\mathrm{NDoH}$. This is evidenced by its major role in the introduction of PCV and RV into the EPI. ${ }^{[8]}$

When there is an intention to add vaccines to the EPI, several factors need to be taken into consideration. These include the burden and significance of the disease within the country, whether there is a safe and effective vaccine available, what the cost-benefit of the vaccine would be and whether the country could afford to implement it, and finally, whether the vaccine could be incorporated practically into the national EPI programme.

\section{Politicall stewardship}

Political leadership in the public health sector became more pronounced in April 2008 when there was a spike in child deaths in the rural Ukhahlamba District (currently Joe Gqabi) of the Eastern Cape Province, with $70 \%$ of 140 deaths reportedly due to gastroenteritis, pneumonia and malnutrition. Rather than any specific disease outbreak, the deaths were mainly attributable to weaknesses in the health system.

Widespread media coverage prompted the Ministry of Health to travel to the Ukhahlamba District to attend to the crisis and offer support. Propelled by the Ukhahlamba deaths, public concern and advocacy efforts to get government to financially and politically commit to the introduction of the vaccines, the Health Minister announced to the 61st World Health Assembly in May 2008 in Geneva, that the vaccines against pneumococcal and rotavirus disease would be introduced within 3 months. Instead of a 2-year lead in, the plan had to be fast-tracked to meet the deadlines, and this had huge financial and programmatic implications as both vaccines would be introduced sooner and simultaneously with the new pentavalent vaccine.

To this day, political commitment is steadfast in all priority programmes.

\section{Programme performance}

The programme indicators are routinely monitored using District Health Information Software (DHIS), whereby the denominators are the Statistics South Africa (StatsSA) mid-year estimates. The denominators for the HPV vaccine are obtained from the Department of Basic Education.

Currently the country has embarked on the Demographic and Health Survey, which will provide immunisation coverage data as one of its deliverables. 
Millestones in EPI in SA

Several milestones have been reached in the history of the EPI in SA:

- 1995: hep B vaccine was introduced

- 1999: Hib vaccine was introduced

- 2000: converted from percutaneous to intradermal route for BCG vaccine

- 2002: neonatal tetanus was eliminated

- 2006: SA was declared polio-free; last reported case was in 1989

- 2008: CPV and RV vaccines were introduced

- 2009: SA first African country and middle-income country to introduce PCV into public immunisation programme, in April (USD20 per dose $=$ ZAR600 million annually $)^{[9]}$

- 2009: SA first African country to introduce RV vaccine into public immunisation programme, in August (USD7.5 per dose = ZAR150 million annually) ${ }^{[9]}$

- 2009: SA switched from whole-cell pertussis vaccine to acellular pertussis vaccine with the introduction of the pentavalent vaccine (5-in-1)

- 2015: SA became the first country in Africa to introduce a fully liquid hexavalent vaccine (6-in-1) by replacing the pentavalent (5-in-1) and hep B vaccines. ${ }^{[9]}$
The EPI is a priority programme of government. It is not going to die because one or two of its officials have resigned from the national office.

1. Bateman C. Vaccines: SA's immunisation programme debunked. S Afr Med J 2016;106(4):318-319. DOI:10.7196/SAMJ.2016.v106i4.10765

2. World Health Organization. Maximising Positive Synergies Collaborative Group: An assessment of interactions between global health initiatives and country health systems. Lancet 2009;373:2137-2169. DOI:10.1016/S0140-6736(09)60919-3

3. Cailhol J, Craveiro I, Madede T, et al. Analysis of human resources for health strategies and policies in 5 countries in sub-Saharan Africa, in response to GFATM and PEPFAR-funded HIV-activities. Globalization Health 2013;9:52.

4. Frenk J, Chen L, Bhutta ZA, et al. Health professionals for a new century: Transforming education to strengthen health systems in an interdependent world. Lancet 2010;376(9756):1923-1958. DOI:10.1016/S0140-6736(10)61854-5

5. Essack S. Human Resources for Health - Challenges and Solutions. Public Health Association of South Africa, 2013. https://www.phasa.org.za/human-resources-for-health-challenges-and-solutions/ (accessed 4 May
2. 2013.

6. Human Resources for Health South Africa: HRH Strategy 2012 to 2017. Pretoria: National Department of Health, 2012. Schoub BD, Mphahlele MJ, Ngcobo NJ, Hoosen AA, Meheus A. Introducing new vaccines into the South African national immunisation programme - a case study. Vaccine 2012;30(Suppl 3):C1-C2 DOI:10.1016/j.vaccine.2012.06.094

8. Baker L. The face of South Africa's Expanded Programme on Immunisation (EPI) schedule. SA Pharmaceutical Journal 2010;Jan/Feb:18-21.

9. Madhi SA. From Vaccine Clinical Trials to Realising Public Health Benefit in South Africa. Presentation to Vaccinology Scientific Congress, National Institute of Communicable Diseases (NICD), Johannesburg, 19 - 20 October 2015.

10. Von Gottberg A, de Gouveia L, Tempia S, et al. Effects of vaccination on invasive pneumococcal disease in South Africa. N Engl J Med 2014;371:1889-1899. DOI:10.1056/NEJMoa1401914

Accepted 26 April 2016 